\title{
Tumour volume as a predictor of metastases in patients presenting with high-grade conventional osteosarcoma of the extremities
}

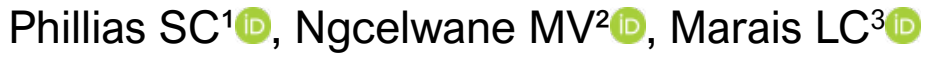 \\ MBChB(Medunsa); Registrar, Department of Orthopaedic Surgery, Steve Biko Academic Hospital, University of Pretoria, Pretoria, South Africa \\ $2 \mathrm{MBChB}(\mathrm{UKZN}), \mathrm{MSc}($ Orth)London, FCS(SA)(Orth); Head of Department of Orthopaedic Surgery, Steve Biko Academic Hospital, University of \\ Pretoria, Pretoria, South Africa \\ 3 MBChB, FC Orth(SA), MMed(Ortho), PhD; Head of Department of Orthopaedic Surgery, School of Clinical Medicine, University of KwaZulu-Natal, \\ Durban, South Africa
}

Corresponding author: Dr SC Phillias, PO Box 191, Siyathuthuka, 1102, South Africa; tel: 079256 1701; email: scphillias@outlook.com

\begin{abstract}
Background: The aim of this study was to compare the initial tumour volume in patients with and without pulmonary and/or skeletal metastases at time of presentation. The secondary aim was to compare the value of tumour volume in the prediction of metastases at time of presentation with known predictive factors, namely serum alkaline phosphatase (ALP) and lactate dehydrogenase (LDH).

Materials and methods: A retrospective cross-sectional analysis was performed comparing the primary tumour volume in patients with and without metastases. All patients with histologically confirmed high-grade conventional osteosarcoma over a five-year period were included.

Results: The study comprised 61 patients. The mean age was 21 years (SD: 11.9, range 5-56) with an equal distribution of males and females $(51 \%$ vs $49 \%)$. There was no correlation between tumour volume and age at presentation $(p=0.31)$. There was no evidence of metastases in only $20 \%(n=12)$ of patients. Skeletal metastases were present in $28 \%(n=16)$ of the patients and pulmonary metastases were present in 44 cases (72\%). There was no significant difference in the tumour volume at presentation between patients with and without pulmonary metastases $(p=0.11)$. However, tumour volume did appear to predict the presence of skeletal metastases $(p=0.02)$. A tumour volume of $1383 \mathrm{~cm}^{3}$ had a negative predictive value (NPV) of $92 \%$ and positive predictive value (PPV) of $55 \%$ for the presence of skeletal metastases (area under curve [AUC] $=0.76$; sensitivity $66 \%$; specificity $87 \%$ ). A tumour volume of $480 \mathrm{~cm}^{3}$ had a $100 \% \mathrm{NPV}$ for the presence of skeletal metastases (AUC=0.74). A tumour volume $\geq 1380 \mathrm{~cm}^{3}$ had an odds ratio $(O R)$ of $13.6(p<0.01$; $95 \% \mathrm{Cl} 2.6-72.5$ ) as an independent variable in relation to skeletal metastases. Multivariate analysis (with ALP and LDH) of tumour volume $\geq 1380 \mathrm{~cm}^{3}$ yielded an OR of $8.6(p=0.04 ; 95 \% \mathrm{Cl} 1.1-67)$ for presence of skeletal metastases.

Conclusion: In this series of conventional high-grade osteosarcoma of the extremities, we found a very high rate of metastases at time of diagnosis. While there was no association with pulmonary metastases, increased tumour volume was associated with an increased risk for the presence of skeletal metastases. More studies in the developing world clinical setting are required to investigate this further; the high rate of metastases seen at time of diagnosis also requires further investigation.
\end{abstract}

Level of evidence: Level 4

Keywords: osteosarcoma, metastases, tumour volume, prognosis, staging

Citation: Phillias SC, Ngcelwane MV, Marais LC. Tumour volume as a predictor of metastases in patients presenting with high-grade conventional osteosarcoma of the extremities. SA Orthop J 2020;19(4):223-228. http://dx.doi.org/10.17159/2309-8309/2020/v19n4a5

Editor: Prof. Theo le Roux, University of Pretoria, Pretoria, South Africa

Received: April 2020

Accepted: August 2020

Published: November 2020

Copyright: $\odot 2020$ Phillias SC. This is an open-access article distributed under the terms of the Creative Commons Attribution Licence, which permits unrestricted use, distribution and reproduction in any medium, provided the original author and source are credited.

Funding: No funding was received for this study.

Conflict of interest: The authors declare that they have no known competing financial interests or personal relationships that could have influenced the work reported in this paper. 


\section{Introduction}

Osteosarcoma is the most common primary malignancy involving bone, excluding myeloma. Nonetheless, it is rare disease, representing less than $1 \%$ of all cancers diagnosed annually in the United States of America and as a result has been classified as rare disease by the World Health Organization. Conventional osteosarcoma is largely a disease of the young, with a second peak of incidence in the elderly. ${ }^{1}$ Surveillance, Epidemiology and End Results (SEER) program data indicated an annual incidence for patients younger than 25 years and older than 60 of 4.4 and 4.2 per million population, respectively. ${ }^{2}$ Osteosarcoma is the fifth most common cancer in adolescents, amounting to more than $10 \%$ of all solid cancers in this age group. ${ }^{3}$

Conventional osteosarcoma, representing approximately $80-90 \%$ of all osteosarcomas, is a high-grade malignancy with a high rate of reported lung metastases, and has a tendency to recur if not completely excised. ${ }^{3,4}$ In developed countries, less than $25 \%$ of patients are diagnosed with metastatic disease at presentation. ${ }^{3}$ In South Africa, patients unfortunately present much later, with previous studies finding metastatic disease in $46-66 \%$ of patients at presentation..$^{5,6}$ Skeletal metastases at the time of diagnosis (socalled synchronous bone metastases) are rare in osteosarcoma and are associated with a poor prognosis. ${ }^{7}$ Furthermore, bones are the first site of subsequent (metachronous) metastases in less than $10 \%$ of cases. ${ }^{8}$

Prior to the advent of chemotherapy, the survival rate, with surgery alone, was extremely poor, with a five-year survival rate of only $10 \% .^{9}$ With current multimodality treatment protocols, which include chemotherapy, survival rates have been found to be in the region of $60-70 \%$ in localised and $20-40 \%$ in metastatic disease. ${ }^{3}$ Several factors have been identified with significant prognostic implications in osteosarcoma including increasing age, the size and site of the primary tumour, serum alkaline phosphatase (ALP) and lactate dehydrogenase (LDH) levels, as well as a poor histological response to neo-adjuvant chemotherapy. Metastatic disease is, however, the single most important predictor of a poor outcome..$^{10}$

Risk factor stratification and the detection of metastases are not only important for accurate prognostic purposes, but also allow early identification of high-risk patients who may require a more aggressive treatment strategy. Early detection of metastases is important as all metastases need to be surgically resected to improve survival, and alternative agents or second-line chemotherapy need to be considered. Therefore, this study sets out to investigate tumour size as a predictor of the presence of metastases at time of presentation in patients with conventional osteosarcoma of an extremity.

\section{Materials and methods}

A retrospective cross-sectional analytical study was performed with data collected at the time of first presentation. Following approval of the study by the relevant ethics boards, records of all patients with osteosarcoma referred to a tertiary level orthopaedic oncology unit in KwaZulu-Natal, South Africa, over five years from 2010 to 2014 were obtained.

Inclusion criteria included diagnosis of osteosarcoma confirmed on biopsy after formal open incisional biopsy, high-grade conventional osteosarcoma histology and osteosarcoma of the extremities. Patients excluded were those with osteosarcoma of the pelvis and axial skeleton, soft tissue osteosarcoma, osteosarcoma variants and surface lesions, where tumour volume or staging data were not available.
Diagnosis of osteosarcoma was confirmed after formal open incisional biopsy was done and subsequently confirmed at a combined radiology-histology meeting. As part of the initial patient workup at presentation, the tumour size was measured on magnetic resonance imaging (MRI), pulmonary metastases on computerised tomography (CT), and skeletal metastases (skip lesions in same bone and distant bone) on technetium bone scan. Due to the retrospective nature of the study, information regarding body weight and height was unavailable, therefore tumour volume was measured using a previously defined formula for an ellipsoidal mass (width $\times$ height $\times$ diameter $\times 0.52$ ). ${ }^{11}$

The demographic characteristics (age and sex of patients, and anatomic location of primary tumour) in patients with and without metastases were recorded. The initial tumour volume on MRI in patients with metastases (skeletal and/or pulmonary) was compared to patients without metastases at time of presentation, i.e. prior to any treatment. The strength of association between tumour volume and the presence of metastases at presentation was then evaluated. We also compared the value of tumour volume in the prediction of metastases at time of presentation with other known predictive factors (serum ALP and LDH).

\section{Statistical analysis}

Data was processed and analysed using Stata 13.0 SE (StataCorp, 2013. Stata Statistical Software: Release 13. College Station, TX: StataCorp LP) and R statistical package 3.0.3 (R Core Team, 2015. R: A language and environment for statistical computing. RFoundation for Statistical Computing, Vienna, Austria). Spearman's correlation test was used to assess the association between patient age and tumour volume. Differences in tumour volume by metastases were assessed using the standard two-sample t-test. Receiver operating characteristic (ROC) curves was used to determine the optimal breakpoint for the classification of metastatic cancer based on tumour volume. The discriminatory power was evaluated by the area under the ROC curve (area under curve or AUC). An AUC value of 0.5 indicates no discriminative ability while an AUC exceeding 0.8 suggests good to excellent predictive capability. Sensitivity and specificity based on the optimal identified cut-points were calculated, along with 95\% confidence intervals (Cl). Logistic regression analysis was employed to estimate the strength of association between tumour volume and metastases. A $p$-value of $<0.05$ was considered statistically significant for all tests.

\section{Results}

Sixty-seven patients were identified with histologically confirmed osteosarcoma involving an extremity. Six patients were excluded from the study. One patient demised prior to completion of systemic staging investigations, and five patients were diagnosed with osteosarcoma variants. Sixty-one patients met the inclusion criteria and their clinical characteristics are provided in Table I. Bone scan was not performed in four patients due to their poor general medical condition which did not allow transfer to the facility where the scan was performed. Ten patients had no tumour volume data available, and were therefore excluded from the tumour-volume analysis.

The mean patient age was 21 years (standard deviation [SD] 11.9 years; range 5-56) and there was an equal distribution between male and female patients ( $51 \%$ vs $49 \%$ ). There was no correlation between tumour volume and age at presentation $(p=0.31)$. The femur $(57 \%)$ and tibia (31\%) were the most commonly involved sites. There was no evidence of metastases in only $20 \% \quad(n=12)$ of the patients. Skeletal metastases were present in $28 \%(n=16)$ of the patients and pulmonary metastases were present in 44 


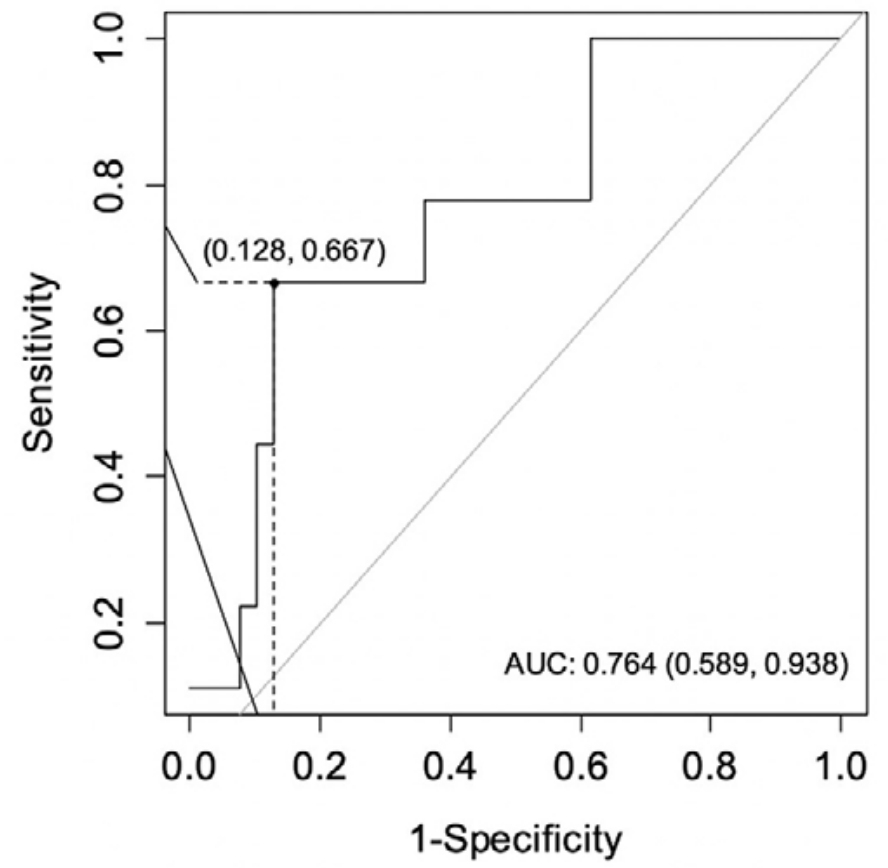

Figure 1(a). Receiver operating characteristic (ROC) curve optimal cutpoint analysis of tumour volume for prediction of skeletal metastases

Table I: Clinical characteristics of cohort

\begin{tabular}{|l|c|c|c|c|}
\hline Characteristic & $\mathbf{n}(\%)$ & Mean & Range & SD \\
\hline Tumour volume $\left.\mathbf{( c m}^{3}\right)$ & 51 & 1114 & $164-6821$ & 1286 \\
\hline $\begin{array}{l}\text { Age (years) } \\
\text { Sex }\end{array}$ & $61(100 \%)$ & 21.3 & $5-56$ & 11.9 \\
\hline Male & $31(51 \%)$ & - & - & - \\
\hline Female & $30(49 \%)$ & - & - & - \\
\hline Site & $35(57 \%)$ & - & - & - \\
\hline Femur & $19(31 \%)$ & - & - & - \\
\hline Tibia & $3(5 \%)$ & & & \\
\hline Fibula & $3(5 \%)$ & - & - & - \\
\hline Humerus & $1(2 \%)$ & - & - & - \\
\hline Ulna & & & & \\
\hline Pulmonary metastases & & & & \\
\hline Yes & $44(72 \%)$ & - & - & - \\
\hline No & $17(28 \%)$ & - & - & - \\
\hline Skeletal metastases & & & & \\
\hline Yes & $16(26 \%)$ & - & - & - \\
\hline No & $41(67 \%)$ & - & - & - \\
\hline Unknown & $4(7 \%)$ & - & - & - \\
\hline
\end{tabular}

cases $(72 \%)$. Of those with skeletal metastases on presentation, $69 \%(n=11)$ had concomitant pulmonary metastases. Five patients with skeletal metastases had more than one bony metastasis, four had single metastases and the number of bony metastases was unknown in seven cases. With respect to pulmonary lesions, 33 patients had multiple lesions, two single metastases, and the number of lesions was unknown in nine cases. The median time to presentation was four months (interquartile range [IQR] 2.5-7, range $1-36$ months, $n=44$ ).

The overall mean tumour volume at presentation was $1114 \mathrm{~cm}^{3}$ (SD $1285 \mathrm{~cm}^{3}$, range 164-6 $821 \mathrm{~cm}^{3}$ ). For patients without any metastases, the mean tumour volume at presentation was $422 \mathrm{~cm}^{3}$

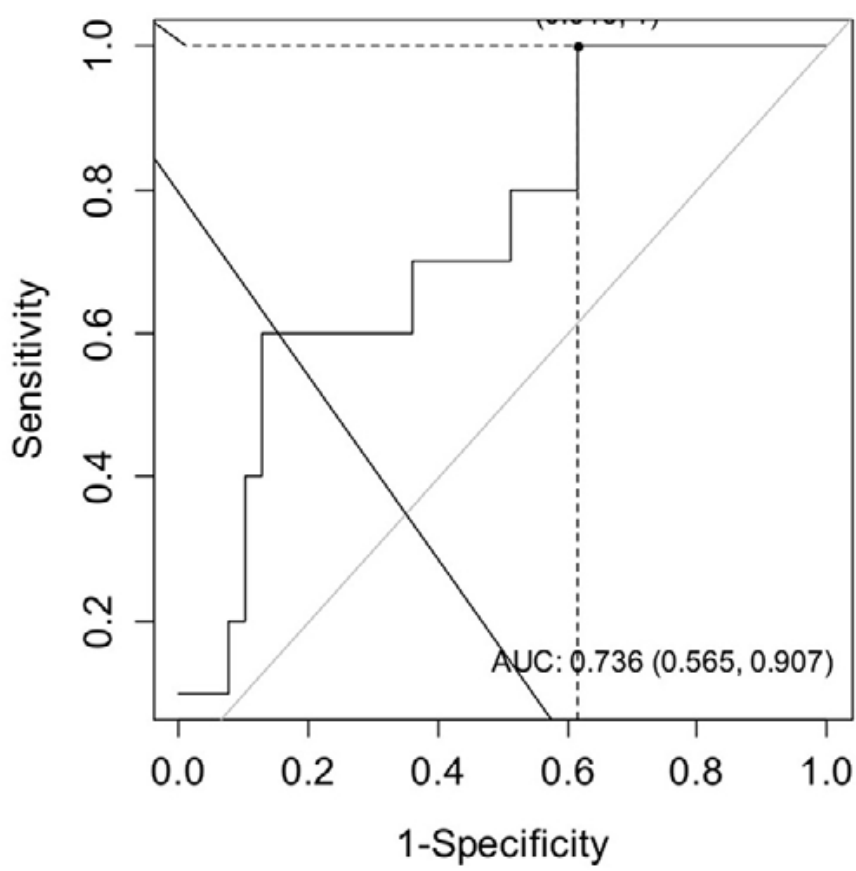

Figure 1(b). Optimal cut-point ROC analysis, optimised for $100 \%$ sensitivity of tumour volume as predictor of skeletal metastases

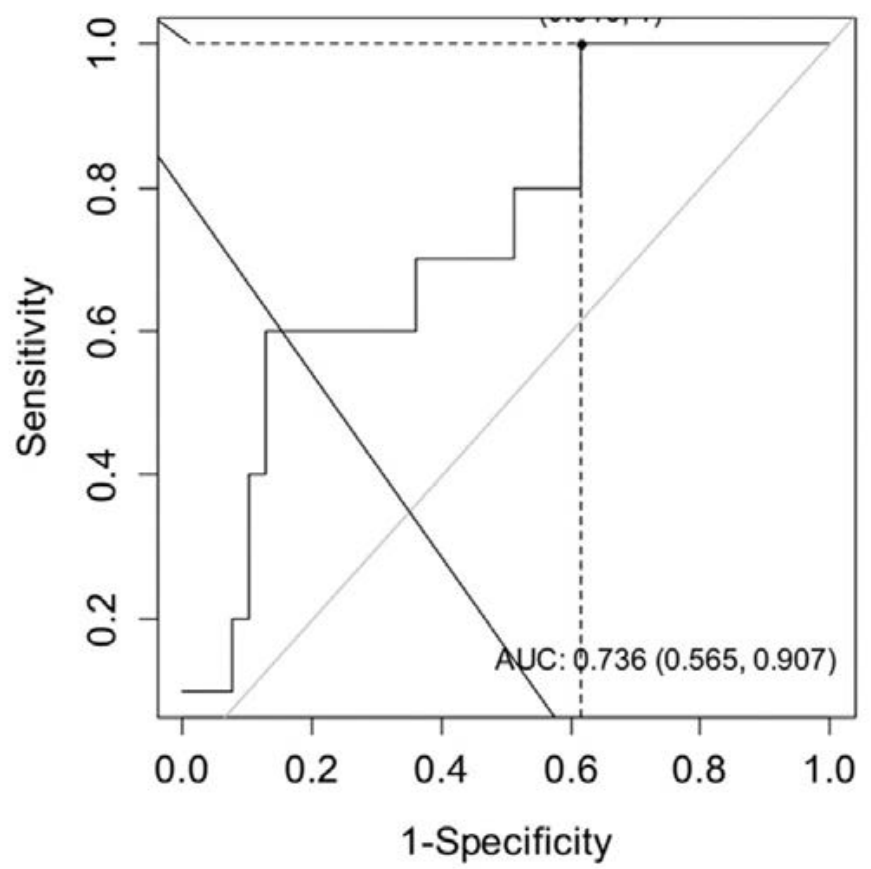

Figure 2. Optimal cut-point ROC analysis optimised for $100 \%$ sensitivity of tumour volume as predictor of skeletal metastases

(range 164-1 $678 \mathrm{~cm}^{3}$ ). The mean tumour volume in patients who presented without pulmonary metastases was $1169 \mathrm{~cm}^{3}(95 \%$ Cl 115-2 $\left.224 \mathrm{~cm}^{3}\right)$ compared to $1093 \mathrm{~cm}^{3}(95 \% \mathrm{Cl} 745-1441$ $\mathrm{cm}^{3}$ ) in patients with pulmonary metastases. The mean tumour volume in patients who presented without skeletal metastases was $829 \mathrm{~cm}^{3}\left(95 \% \mathrm{Cl} 523-1136 \mathrm{~cm}^{3}\right)$ compared to $2016 \mathrm{~cm}^{3}(95 \% \mathrm{Cl}$ $487-3545 \mathrm{~cm}^{3}$ ) in patients with skeletal metastases. Analysis of the association between tumour volume and metastases showed that there was no significant difference in the tumour volume at presentation between patients with and without pulmonary metastases $(p=0.851)$. However, tumour volume did appear to predict the presence of skeletal metastases $(p=0.010)$. 
ROC analysis (Figure 1) was then used to identify the optimal cut-off volumes to predict the presence of metastases. A tumour volume of $1383 \mathrm{~cm}^{3}$ had a negative predictive value (NPV) of $92 \%$ and positive predictive value (PPV) of $55 \%$ for the presence of skeletal metastases (AUC=0.76; sensitivity $66 \%$; specificity $87 \%$ ). When optimising to achieve a $100 \%$ sensitivity (Figure 2), a tumour volume of $480 \mathrm{~cm}^{3}$ had a $100 \%$ NPV for the presence of skeletal metastases (AUC=0.74).

Using univariate logistic regression to assess the strength of association of the tumour volume $\geq 1380 \mathrm{~cm}^{3}$ as an independent variable in relation to skeletal metastases, an odds ratio (OR) of $13.6(p<0.01 ; 95 \% \mathrm{Cl} 2.6-72.5)$ was identified. Multivariate analysis (with ALP and LDH) of tumour volume $\geq 1380 \mathrm{~cm}^{3}$ yielded an OR of $8.6(p=0.04 ; 95 \% \mathrm{Cl} 1.1-67)$ for presence of skeletal metastases, (Table II).

\section{Discussion}

Osteosarcoma is the most frequent malignant bone tumour in paediatric patients. ${ }^{12}$ Several prognostic factors have been proposed including detectable metastases, advanced age, non-extremity locations, large tumour volume, elevated LDH or ALP, and poor histological response to chemotherapy. Of these, metastatic disease, large tumour sizes and poor response to neoadjuvant chemotherapy have consistently been associated with a poor outcome. ${ }^{13}$ Tumour size may reflect the tumour burden and/or the extent of disease. A large primary tumour is more likely to be associated with distant metastases. ${ }^{14}$ Furthermore, tumour size has been associated with an increase in risk of mortality. Patients with tumours of diameter of $>15 \mathrm{~cm}$ have a three-fold higher risk of death, whereas with tumour diameters of $<15 \mathrm{~cm}$ survival is better. ${ }^{15}$

There is a paucity of literature relating to tumour volume as a prognostic indicator from low- to middle-income countries. In addition, few studies have previously studied the relationship between tumour volume and the presence of lung and skeletal metastases, independently. Furthermore, there is a lack of data relating to the cut-off values, in terms of tumour volume, for presence of metastases. A previous study, for example, only looked at cut-off values for tumour volume in predicting lung metastases and excluded skeletal metastases. ${ }^{14}$ With this study we aimed to assess the association of tumour volume with pulmonary and skeletal metastases in a developing world setting.

In our study, the majority of patients presented with relatively large tumours, with a mean tumour volume of $1114 \mathrm{~cm}^{3}$. Tumour size $>10 \mathrm{~cm}$ in length has previously been shown as an adverse prognostic factor for overall survival. ${ }^{16}$ Another multivariate model incorporating factors predicting treatment failure also found patients with large tumour size (>12 cm in length) to have a statistically significant worse prognosis for survival. ${ }^{12}$ In South Africa, patients often present with advanced disease, with previous studies reporting metastatic disease in $46 \%$ to $66 \%$ of patients at time of diagnosis. ${ }^{5,6} \mathrm{~A}$ previous study from South Africa also found advanced stage of disease at presentation, with $48 \%$ of cases having detectable metastases at time of presentation. ${ }^{6}$ In their series, only $28 \%$ of tumours were $<10 \mathrm{~cm}$ in length. The authors concluded that the presence of metastases at diagnosis and size $>10 \mathrm{~cm}$ in length were associated with a poor prognosis.

Our study found a considerably higher incidence of clinically detectable metastatic disease at initial presentation when compared to expected rates in developed countries (10-20\%). ${ }^{17}$ In particular, the high rate $(28 \%)$ of skeletal metastases at time of presentation is noteworthy. It remains unclear if the high rate of metastases seen in our series is related to a delay in diagnosis or if it might be related to a more aggressive phenotype of osteosarcoma. A pooled
Table II: Multivariate analysis of risk factors for the presence of skeletal metastasis at time of diagnosis

\begin{tabular}{|l|c|c|c|}
\hline & Odds ratio & $95 \% \mathbf{C l}$ & p-value \\
\hline $\mathrm{LDH} \geq 850 \mathrm{IU} / \mathrm{L}$ & 2.7 & $0.36-20.04$ & 0.330 \\
\hline $\mathrm{ALP} \geq 280 \mathrm{IU} / \mathrm{L}$ & 9.8 & $1.35-70.87$ & 0.024 \\
\hline $\begin{array}{l}\text { Tumour volume } \\
\geq 1380 \mathrm{~cm}^{3}\end{array}$ & 8.7 & $1.11-67.18$ & 0.039 \\
\hline
\end{tabular}

analysis by Marko et al. found the highest prevalence of metastases at diagnosis of osteosarcoma in countries with a medium or low Human Development Index (HDI). ${ }^{17}$ The prevalence of metastatic osteosarcoma at diagnosis in very high $\mathrm{HDI}$, high $\mathrm{HDI}$, and medium or low HDI groups was found to be $15 \%, 20 \%$ and $31 \%$, respectively. They suggested that socioeconomic status, educational levels, as well as healthcare system and resource constraints in countries with medium/low HDI may result in a treatment delay with resulting increase in the rate of metastases at diagnosis. ${ }^{17}$ Irrespective of the cause, these findings are particularly relevant in the South African clinical setting and again highlight the need for early referral of cases to specialised orthopaedic oncology units.

It has been noted that patients who present with metastases have a shorter interval between onset of symptoms and diagnosis. ${ }^{18}$ This finding is somewhat counter-intuitive and suggests that aggressive biologic behaviour may be more important in the pathogenesis of metastases than delay in diagnosis. Tumour volume at time of presentation may also be similarly difficult to interpret and patients with smaller tumours do not necessarily have a lower rate of metastases. Initial tumour volume has, however, been shown to be of high prognostic value, and $150 \mathrm{~cm}^{3}$ tumour volume has been proposed as the cut-off point in predicting relapse and the development of metastases. ${ }^{19}$ Kaste et al. found a median tumour volume of $717 \mathrm{~cm}^{3}$ in patients with lung nodules at diagnosis. ${ }^{20}$ Due to the wide range of values $\left(63-3520 \mathrm{~cm}^{3}\right)$ in the 28 patients with metastases at diagnosis, the authors were, however, unable to correlate the primary tumour volume with the presence of metastases or overall survival. Bacci et al. found the incidence of metastases to be $20 \%$ and $12 \%$ in patients with tumour volumes $>150 \mathrm{ml}$ and $<150 \mathrm{ml}$, respectively. Tumour volume $>150 \mathrm{ml}$, however, remained a significant predictor of the presence of metastases following multivariate logistic regression analysis. ${ }^{18}$ In our series, tumour volume was not a significant predictor for the presence of pulmonary metastases at diagnosis. Munajat et al. previously looked at the association between tumour volume and lung metastases; $47 \%$ of their patients had evidence of lung metastases at presentation. ${ }^{14}$ They found a significant difference in primary tumour volumes in patients with and without metastases. Their cut-off value of tumour volume was at $371 \mathrm{~cm}^{3}$. Munajat et al. did not report on skeletal metastases. ${ }^{14}$ While we found no association with pulmonary metastases, we found that tumour volume was associated with the presence of skeletal metastases in our series.

The lung remains the most common site for metastases in osteosarcoma, with only about $10 \%$ of cases reported to develop skeletal metastases. ${ }^{21}$ Skeletal metastase have also been associated with a particularly poor survival. ${ }^{22}$ The 2014 European Society of Musculoskeletal Oncology (ESMO) guidelines recommend that all patients undergo a technetium bone scan during staging to search for the presence of skeletal metastases. ${ }^{23}$ In general, the aim is to conclude all staging investigations as soon as possible so as to not delay the treatment of the malignancy. In resource-poor developing countries like South Africa, however, it might be difficult to obtain nuclear imaging studies, or in some cases, waiting times may be exceedingly long. Thus, it may be useful to identify other markers 
that can be used to confirm or exclude the presence of skeletal metastases in patients with osteosarcoma.

In this cohort, univariate analysis showed that patients with a tumour volume $\geq 1380 \mathrm{~cm}^{3}$ had a 13 times higher risk of having skeletal metastases at the time of diagnosis. In a multivariate model with ALP and LDH, the OR decreased to 8.6 but the association remained significant. ROC analysis revealed that a tumour volume cut-off value of $1383 \mathrm{~cm}^{3}$ yielded a $92 \%$ NPV for skeletal metastases. The absence of skeletal metastases could be predicted with a sensitivity of $100 \%$ by reducing this value to $480 \mathrm{~cm}^{3}$.

Our findings suggest that, in this series, patients with a tumour volume below $480 \mathrm{~cm}^{3}$ were highly unlikely to have clinically detectable skeletal metastases at time of diagnosis. Furthermore, patients with a tumour volume higher than $1380 \mathrm{~cm}^{3}$ at time of presentation have an increased risk of skeletal metastases. Consideration could be given to the use of more sensitive screening investigations in patients with such large tumours, for example. The sensitivity and specificity of bone scintigraphy for detection of bone metastases is $78 \%$ and $48 \%$, respectively. ${ }^{24}$ Recently, ${ }^{18} \mathrm{~F}$-fluorodeoxy-D-glucose positron emission tomography $\left({ }^{18} \mathrm{~F}-\mathrm{FDG}\right.$ $\mathrm{PET}$ ) and positron emission tomography with computed tomography (PET CT) has emerged as a useful investigation to identify skeletal lesions. ${ }^{24} \mathrm{~A}$ meta-analysis by Liu et al. demonstrated a sensitivity of $93 \%$ and specificity of $97 \%$ for ${ }^{18} \mathrm{~F}-\mathrm{FDG}$ PET and PET CT. ${ }^{25}$

While these findings are interesting, there are numerous shortcomings to this study which need to be considered. First, the small sample size makes any definitive recommendation in this regard impossible. This is evident when looking at the wide $95 \% \mathrm{Cl}$ in our multivariate regression analysis. Secondly, it would have been ideal to correlate tumour volume not only with presence of metastases but also overall survival. A large number of patients were, however, lost to follow-up, which precluded longitudinal prognostication. The major confounding factor is that there are numerous factors that have an influence on the prognosis of osteosarcoma and our multivariate model may not have been sufficiently robust to exclude the association of other factors with the presence of skeletal metastases at time of diagnosis. Due to the retrospective nature of the study, data on the time to presentation and the specific histological subtype were not uniformly available. These measurements should be included in future studies as they have important bearing on the rate of metastases and overall patient survival.

Larger, well-designed studies with long-term follow-up are thus needed to determine the association of tumour volume with the risk of pulmonary and skeletal metastases in the developing world setting. Further research is also needed to investigate the high incidence of metastases, and skeletal metastases in particular, at time of presentation.

\section{Conclusion}

In this series of conventional high-grade osteosarcoma of the extremities, we found a very high rate of metastases at time of diagnosis. While there was no association with pulmonary metastases, increased tumour volume was associated with an increased risk for the presence of skeletal metastases. More studies in the developing world clinical setting are required to investigate this further; the high rate of metastases seen at time of diagnosis also requires further investigation.

\section{Acknowledgements}

We would like to thank Ms Tshifhiwa Nkwenika from the South African Medical Research Council, Biostatistics Research Unit in Pretoria, and Prof. Benn Sartorius for their input on the statistical aspects of the study.

\section{Ethics statement}

All procedures performed in studies involving human participants were in accordance with the ethical standards of the institutional and/or national research committee and with the 1964 Helsinki declaration and its later amendments or comparable ethical standards. For this study formal consent was not required. The database from which eligible patients were identified received ethical approval from the UHERB ethics review board (ref no. 02-012013). Further ethical approval for the study was obtained from the University of Pretoria Ethics Board (ref no. 585/2018).

\section{Declaration}

The authors declare authorship of this article and that they have followed sound scientific research practice. This research is original and does not transgress plagiarism policies.

\section{Author contributions}

SCP: Primary author, responsible for data collection and interpretation, manuscript preparation and revision.

MVN: Study supervisor, responsible for manuscript preparation and revision.

LCM: Study supervisor, responsible for conceptualisation and study design, data collection; assisted with statistical analysis and interpretation, manuscript preparation and revision.

\section{ORCID}

Phillias SC (D) https://orcid.org/0000-0001-7452-5721

Ngcelwane MV (D) https://orcid.org/0000-0001-7564-3308

Marais LC (iD) https://orcid.org/0000-0002-1120-8419

\section{References}

1. Dean BJF, Whitwell D. (i) Epidemiology of bone and soft-tissue sarcomas. Orthopaedics and Trauma. 2009;23(4):223-30. https:// doi.org/10.1016/j.mporth.2009.05.006.

2. Mirabello L, Troisi RJ, Savage SA. Osteosarcoma incidence and survival rates from 1973 to 2004: Data from the Surveillance, Epidemiology and End Results Program. Int $J$ Cancer. 2009;115(7):1531-43. https://doi.org/10.1002/cncr.24121.

3. Hogendoorn PCW, Athanasou N, Bielack S, et al. Bone sarcomas: ESMO Clinical Practice Guidelines for diagnosis, treatment, and follow-up. Ann Oncol. 2010;21(Supplement 5):v204-v213. https:// doi.org/10.1093/annonc/mdq223.

4. Kim HJ, Chalmers PN, Morris CD. Pediatric osteogenic sarcoma. Curr Opin Pediatr. 2010;22:61-66. https://doi.org/10.1097/ MOP.0b013e328334581f.

5. Ferriera N, Marais LC. Osteosarcoma presentation stages to a tumour unit in South Africa. S Afr Med J. 2012;102(8):673-76. https://doi.org/10.7196/SAMJ.5835.

6. Shipley JA, Beukes CA. Outcomes of osteosarcoma in a tertiary hospital. S Afr Orthop J. 2012;11(1):18-22.

7. Kager L, Zuobek A, Kastner U, et al. Skip metastases in osteosarcoma: Experience of the Cooperative Osteosarcoma Study Group. J Clin Oncol. 2006;24:1535-41.

8. Bacci G, Ferrari S, Longhi A, et al. Pattern of relapse in patients with osteosarcoma of the extremities treated with neoadjuvant chemotherapy. Eur J Cancer. 2001;37:32-38.

9. Campanacci M, Bacci G, Bertoni $F$, et al. The treatment of osteosarcoma of the extremities: Twenty years' experience at the Istituto Ortopedico Rizzoli. Cancer. 1981;48:1569-81. https://doi.org/10.1002/1097-0142(19811001)48:7<1569::AIDCNCR2820480717>3.0.CO;2-X.

10. Stokkel MPM, Linthorst MFG, Borm JJJ, Taminiau AH, Pauwels EKJ. A reassessment of bone scintigraphy and commonly tested pretreatment biochemical parameters in newly diagnosed osteosarcoma. J Cancer Res Clin Oncol. 2002;128:393-99. https:// doi.org/10.1007/s00432-002-0350-5.

11. Shin $\mathrm{KH}$, Moon SH, Suh JS, Yang WI. Tumor volume change as a predictor of chemotherapeutic response in osteosarcoma. Clin Orthop Relat Res. 2000;376:200-208. https://doi. org/10.1097/00003086-200007000-00027.

12. Vasquez L, Tarrillo F, Oscanoa M, et al. Analysis of prognostic factors in high-grade osteosarcoma of the extremities in children: a 15-year single-institution experience. Front Oncol. 2016;6:22. https://doi.org/10.3389/fonc.2016.00022.

13. Ahmad O, Soodeh A, Ali M. Osteosarcoma: Current concepts, challenges, and future directions. Curr Orthop Pract. 
2015;26(2):181-98. https://doi.org/10.1097/BCO.00000000000 00199.

14. Munajat WZ, Norazman MZ, Wan Faisham WI. Tumour volume and lung metastasis in patients with osteosarcoma. $J$ Orthop Surg. 2008;16(2):182-85. https://doi.org/10.1177/230949900801600211.

15. Petrilli AS, De Camargo B, Filho VO, et al. Results of the Brazilian Osteosarcoma Treatment Group Studies III and IV: prognostic factors and impact on survival. J Clin Oncol. 2006;24:1161-68. https://doi.org/10.1200/JCO.2005.03.5352.

16. Berner K, Hall KS, Monge OR, et al. Prognostic factors and treatment results of high-grade osteosarcoma in Norway: A scope beyond the 'classical' patient. Sarcoma. 2015;3:1-14. https://doi. org/10.1155/2015/516843.

17. Marko A, Diessner BJ, Spector LG. Prevalence of metastasis at diagnosis of osteosarcoma: an international comparison. Pediatr Blood Cancer. 2016;63:1006-11. https://doi.org/10.1002/ pbc.25963.

18. Bacci G, Ferrari S, Longhi A, et al. High-grade osteosarcoma of the extremity: differences between localized and metastatic tumors at presentation. J Pediatr Hematol Oncol. 2002;24:27-30. https://doi. org/10.1097/00043426-200201000-00008.

19. Bieling $P$, Rehan N, Winkler $P$, et al. Tumor size and prognosis in aggressively treated osteosarcoma. J Clin Oncol. 1996;14(3):84858. https://doi.org/10.1200/jco.1996.14.3.848 .

20. Kaste SC, Pratt CB, Cain AM, Jones-Wallace DJ, Rao $B N$. Metastases detected at the time of diagnosis of primary pediatric extremity osteosarcoma at diagnosis. Cancer. 1999;86(8):1602-08. https://doi.org/10.1002/ (SICl)1097-0142(19991015)86:8<1602::AID-CNCR31>3.0.CO;2-R.

21. Bacci $G$, Longhi $A$, Bertoni $F$, et al. Bone metastases in osteosarcoma patients treated with neoadjuvant or adjuvant chemotherapy: The Rizzoli experience in 52 patients. Acta Orthop. 2006;77(6):938-43. https://doi.org/10.1080/17453670610013268.

22. Ferrari S, Briccoli $A$, Mercuri $M$, et al. Postrelapse survival in osteosarcoma of the extremities: prognostic factors for long-term survival. J Clin Oncol. 2003;21:710-15. https://doi.org/10.1200/ JCO.2003.03.141.

23. ESMO/European Sarcoma Networking Group. Bone sarcomas: ESMO Clinical Practice Guidelines for diagnosis, treatment and follow-up. Ann Oncol. 2014;25(S3):iii113-iii123. https://doi. org/10.1093/annonc/mdu256.

24. O'Sullivan GJ, Carty FL, Cronin CG. Imaging of bone metastasis: An update. World J Radiol. 2015;7(8):202-11. https://doi. org/10.4329/wjr.v7.i8.202.

25. Liu F, Zhang Q, Zhiu D, Dong J. Effectiveness of ${ }^{18} \mathrm{~F}-\mathrm{FDG}$ PET/CT in the diagnosis and staging of osteosarcoma: a meta-analysis of 26 studies. BMC Cancer. 2019:323. https://doi.org/10.1186/ s12885-019-5488-5. 